\title{
Differentiation of the 4D MRI blood flow data to estimate the vorticity and shear stress in aorta, pulmonary artery and the heart
}

\author{
Igor Nesteruk \\ Institute of Hydromechanics, NASU \\ Igor Sikorsky Kyiv Polytechnic \\ Institute, Kyiv, Ukraine \\ inesteruk@yahoo.com
}

\author{
Filippo Piatti \\ $3 D$ and Computer Simulation \\ Laboratory, IRCCS Policlinico San \\ Donato, San Donato Milanese, Italy \\ filippo.piatti@grupposandonato.it \\ Alberto Redaelli \\ Dipartimento di Elettronica, \\ Informazione e Bioingegneria \\ Politecnico di Milano \\ Milan, Italy \\ alberto.redaelli@polimi.it
}

\author{
Dmytro Sytnyk \\ Numerical Mathematics Dpt. \\ Institute of Mathematics, National \\ Academy of Sciences of Ukraine \\ Kyiv, Ukraine \\ sytnikd@gmail.com
}

\begin{abstract}
Magnetic resonance imaging (MRI) using threedimensional velocity encoding phase contrast methods offers the opportunity to quantify time-resolved 3D flow patterns in vivo. This technique can have a breakthrough impact on the evaluation, risk stratification and surgical planning in hemodynamic-related pathologies, e.g., cardiac valve diseases, arterial stenos or insufficiency, dilation, dissection or coartaction. Nevertheless, the limited time and space resolution of 4D MRI data restricts the accuracy of derivative calculations, which are necessary for the estimation of some very important characteristics such as vorticity and shear stress in the blood flow. In this paper we introduce a new accuracy measure for the shear stress and calculate the possible discrepancy with the use of a new software tool which automatically segment the vessels and provides the information along the center line, between two selected cross-sections and in the vicinity of the selected points. Results are provided both as maximum and time-averaged values in aorta, pulmonary artery, left and right ventricles and valves. The cavitation phenomena, revealed in artificial mitral heart valves, cause interest in such studies for the real valves, especially for the patients suffering from different cardio-vascular diseases. To estimate possible cavitation inception the vorticity and pressure drop in mitral valve were estimated.
\end{abstract}

Keywords-Magnetic resonance imaging; 4D MRI; blood flow characteristics, cavitation inception

\section{INTRODUCTION}

Magnetic resonance imaging (MRI) using threedimensional velocity encoding phase contrast methods offers the opportunity to quantify time-resolved 3D blood flow patterns in vivo, e.g., [1-7]. This novel imaging technique allowed unraveling most of the fluid-dynamic patterns inside big vessels (e.g., aorta) and heart chambers (ventricles, atria), both in physiological and pathological conditions. Besides giving important quantitative and qualitative information on in vivo blood flow, 4D Flow MRI data is characterized by limited time and space resolution, hampering the estimation of some important markers (e.g., vorticity, shear stresses) due to inaccurate velocity derivatives computations. Such hemodynamic markers have been linked to degenerations of the endothelial tissue in bicuspid aortic valve diseases [8], as well as to right ventricle diastolic disfunction [9], in searching for novel predictors of pathology worsening. However, no reliable methods to understand the accuracy of shear stresses estimation in big vessels (e.g., aorta, pulmonary arteries) have been yet proposed in literature.

Also, in vivo blood flow can be determinant in assessing peculiar hemodynamic features or patterns, such as cavitation phenomena. In particular, theoretical studies of this phenomenon can be found in [10-12]. Cavitation assumes a role in depicting non-physiological blood flow due to separation regions and vortex formation. Indeed, the cavitation phenomena was revealed in artificial mitral heart valves (or mechanical heart valves (MHV)) with the use of Doppler ultrasonography (e.g., [10]) and the corresponding signals were registered during the valve closure. In [10] the photographs of the cavitation bubbles downstream of a closing mitral MHV were obtained. In [13] the pressure fluctuations were measured in a left ventricular assist device, imbedded into a cow body, and it was shown that a pressure drop occurs, which is sufficient for the cavitation inception. One of the reasons for cavitation to occur was assumed to be the formation of vortexes, which low core-region pressure allows this phenomenon to occur. Thus, an accurate quantification of vorticity and vortex region can indicate possible cavitation phenomena. By contrast, no cavitation was revealed in natural human heart valves possibly due to the compliance of the natural leaflets, which reduces the pressure drop and makes the cavitation impossible, [10-12]. According to these evidences and assumptions, the analysis of flow peculiarities in the heart chambers may be pivotal to the understanding of such phenomena; in such a way, 4D Flow could become a novel tool to visualize and quantify in vivo the performances of artificial devices, with respect to physiological blood flow patterns. Importantly, no studies have been reported to unravel cavitation phenomena from in vivo blood flow in heart chambers.

In such a context, in this paper we introduce a novel software tool developed to process 4D Flow data both in big vessels and heart chambers, thus allowing us to visualize and quantify blood flow features during the cardiac cycle. In particular, we introduced new accuracy measure for the shear stress and calculate the possible discrepancy with respect to the local direction of the vessel, in order to define the quality of shear stress computation with a quantitative measure of inaccuracy. Also, we proposed a method to estimate possible cavitation inception in heart chambers from accurate vorticity measures. 


\section{MATERIALS AND MEthodS}

\section{A. 4D MRI Data Sets}

We used phase contrast MRI data (see, e.g., [1]), which provides time dependent 3 components of the blood flow velocity with the space resolution of approximately $2 \mathrm{~mm}$ and time resolution of 10-20 time frames during one heart bit. These characteristics ensure large enough number of points in the aorta, pulmonary artery and the heart in order to segment the lumen and to analyze the flow patterns. We analyzed 25 datasets, which can be divided into three groups: A - healthy volunteers, B - Bicuspid aortic valve (BAV) without visible changes in the aorta shape and blood flow, $\mathrm{C}$ - BAV patients with the aorta dilation and severe pathological hemodynamic changes. We use MATLAB software for post-processing DICOM files, developing the codes and creating a user-friendly interface.

\section{B. Estimations of the Wall Shear Stress and Vorticity Based} on the Peculiarities of the Steady Hagen-Poiseuille Flow in Cylindrical Pipes

We used the properties of the steady Hagen-Poiseuille flow in the cylindrical pipe of radius $R$ (see, e.g., [14]) to segment the vessels and to estimate the wall shear stress and vorticity. The local velocities of this flow are directed along the pipe axis and its magnitude $U$ depends on the radial distance from the axis $r$, dynamic viscosity coefficient $\mu$ (we will use the value $3 \mathrm{cP}$ for blood) and the pressure gradient $d p / d x$ along the pipe axis.

$$
U=-\frac{1}{4 \mu} \frac{d p}{d x}\left(R^{2}-r^{2}\right)
$$

We used the mean velocity $u$ averaged at a cross section of the pipe

$$
u=\frac{2}{R^{2}} \int_{0}^{R} r U d r=-\frac{R^{2}}{8 \mu} \frac{d p}{d x}
$$

Then the pressure gradient along the pipe axis is expressed as follows:

$$
\frac{d p}{d x}=-\frac{8 \mu \pi u}{S}=-\frac{8 \mu \pi Q}{S^{2}}
$$

Where $S$ is the cross sectional area, $Q$ is the volumetric flow rate. Knowing the pressure gradient, the wall shear stress was estimated with the use of formulae (1)-(3):

$$
\tau=-\left.\mu \frac{d U}{d r}\right|_{r=R}=-\frac{R}{2} \frac{d p}{d x}=\frac{4 \mu u}{R}=\frac{4 \mu Q}{\pi R^{3}}
$$

Formulae (1) and (3) allow calculating the blood flow vorticity vector $\bar{\omega}=\operatorname{rot}(\bar{U})$ and its magnitude:

$$
|\bar{\omega}|=\frac{r}{2 \mu} \frac{d p}{d x}=\frac{4 u r}{R^{2}}
$$

Thus, in the steady Hagen-Poiseuille flow the maximal vorticity occurs on the pipe walls (at $r=R$ ) and is equal

$$
|\bar{\omega}|_{\max }=\frac{4 u}{R}
$$

\section{Lumen Segmentation and Calculations of the Parameters along the Center Line.}

The algorithm of the segmentation is based on the fact that blood flow can be assumed as laminar and similar to (1) at some moments of the cardiac cycle or when the timeaveraged velocities are used, [4, 5]. The algorithm implemented is based on an automatized process, only requiring one point $(\mathrm{Pi})$ to be selected inside the vessel. Then, the nearest point of the MRI grid is identified as the closest voxel to Pi. Given that in a vessel cross-section all velocities are mostly directed along its normal (see (1)), the corresponding cross-section, the nearest points of the grid with the similar directions of the velocity was found and the center of the cross-section can be calculated. The next point inside the vessel can be calculated by a small (one voxel) shift along the normal from the center of the cross-section (up- or downstream of the blood flow). By repeating the previous procedure, all points of MRI grid located inside the vessel can be extracted and the characteristics of the blood flow can be calculated. Similar to $[4,5]$, we will use the time averaged data and the instant velocity components to segment the vessels.

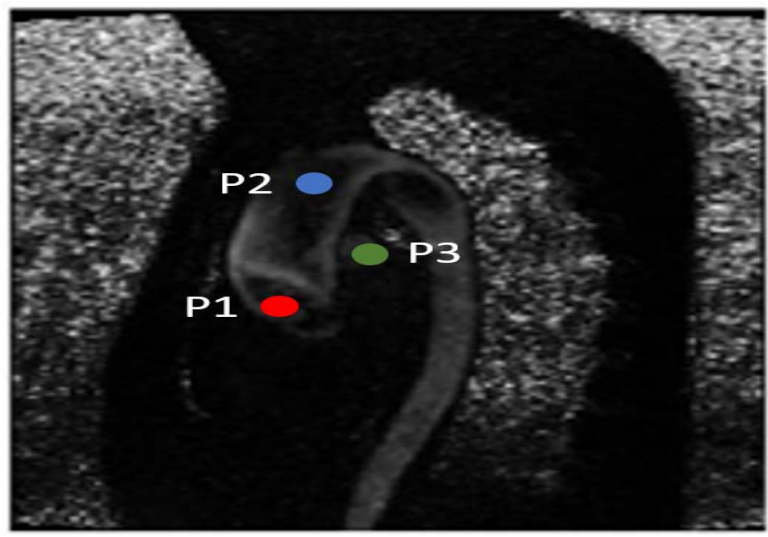

a)

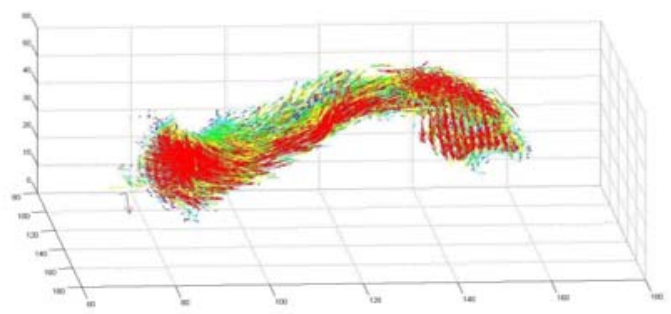

b) 


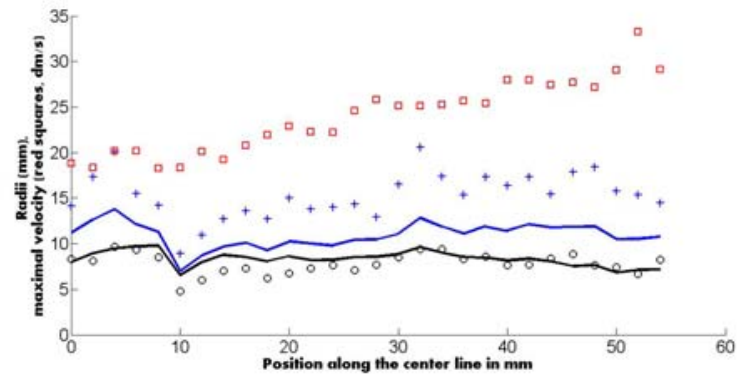

c)

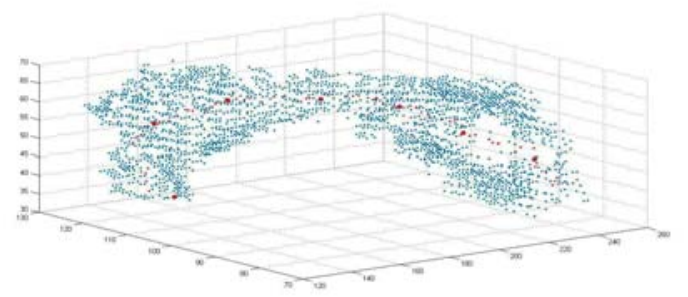

d)

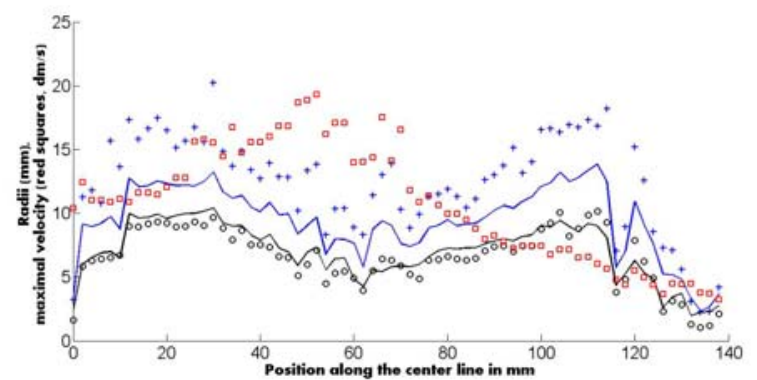

e)

Fig. 1. Segmentation of the aorta, pulmonary artery and right ventricle with the use of the automatic algorithm $([4,5])$. Only one point was selected manually (red and green points $\mathrm{P} 1$ and $\mathrm{P} 3$ in a)). To visualize only a part of the artery another point can be fixed (see blue point P2 located inside the aorta in a)). The blood flow in aorta at a moment of maximum systole is shown in Fig. 1b. The cross-sectional radii and maximum velocities (red "squares") distributions along the center lines are shown in Figs. 1c and 1e. Blue crosses show the maximal radius of the cross-section, other lines and markers correspond to the different averaged values. Fig. 1d represents the segmented shape of the pulmonary artery and right ventricle. All coordinates in b) and d) are in $\mathrm{mm}$.

Fig. 1 illustrates the segmentation of the aorta (between two selected red (P1) and blue (P2) points), the pulmonary artery and the right ventricle (upstream to the selected green point P3) for a patient from the $\mathrm{C}$ group. It can be seen that the maximal radius can be twice grater as the averaged ones. The maximal blood flow speeds are very high in this patient both in aorta and in the pulmonary artery (in healthy patients the maximal velocity is approximately twice smaller).

\section{Differentiation of the Velocity Field}

To calculate the geometrical and flow parameters presented in Figs. 1c and 1e, there is no need to differentiate the velocity field. Nevertheless, some important local characteristics (e.g., shear stress and vorticity) need calculations of the derivatives. In particular, shear stress at the plane with the normal vector $\bar{n}=\left(n_{1}, n_{2}, n_{3}\right)$ (see Fig. 2) can be calculated as follows:

$$
\vec{\tau}_{n}=\mu\left(\begin{array}{l}
2 n_{1} \frac{\partial V_{x}}{\partial x}+n_{2}\left(\frac{\partial V_{x}}{\partial y}+\frac{\partial V_{y}}{\partial x}\right)+n_{3}\left(\frac{\partial V_{x}}{\partial z}+\frac{\partial V_{z}}{\partial x}\right) \\
n_{1}\left(\frac{\partial V_{x}}{\partial y}+\frac{\partial V_{y}}{\partial x}\right)+2 n_{2} \frac{\partial V_{y}}{\partial y}+n_{3}\left(\frac{\partial V_{y}}{\partial z}+\frac{\partial V_{z}}{\partial y}\right) \\
n_{1}\left(\frac{\partial V_{x}}{\partial z}+\frac{\partial V_{z}}{\partial x}\right)+n_{2}\left(\frac{\partial V_{y}}{\partial z}+\frac{\partial V_{z}}{\partial y}\right)+2 n_{3} \frac{\partial V_{z}}{\partial z}
\end{array}\right)
$$

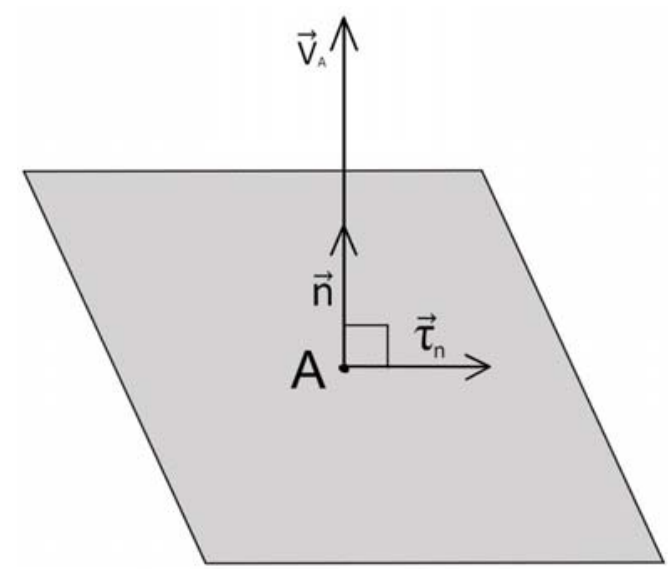

Fig. 2. Plane, normal vector and shear stress.

The derivatives presented in formula (7) can be approximated with the use of values in the neighboring points. E.g., a derivative at a red point shown in Fig. 3 can be estimated as follows:

$$
\frac{\partial V_{x}}{\partial x} \approx \frac{1}{2}\left(\frac{V_{+1}-V_{0}}{h_{x}}+\frac{V_{0}-V_{-1}}{h_{x}}\right)=\frac{V_{+1}-V_{-1}}{2 h_{x}}
$$

Where $V_{+1}$ and $V_{-1}$ are the velocity components on the next and previous levels respectively, which can be taken without any smoothing (the values corresponding to the red squares only) - first method. We can put also the average values on corresponding levels (all red, blue and green points) with the use of constant weighing coefficient $1 / 9$ - second method; or use the weighing coefficients shown in Fig. 3 - third method, Sober filter [15]. To calculate the derivatives in (7) we will use also the fourth method - a corrected Sober filter, taking into account that in incompressible fluid holds the equation:

$$
\operatorname{div}(\vec{V}) \equiv \frac{\partial V_{x}}{\partial x}+\frac{\partial V_{y}}{\partial y}+\frac{\partial V_{z}}{\partial z}=0
$$




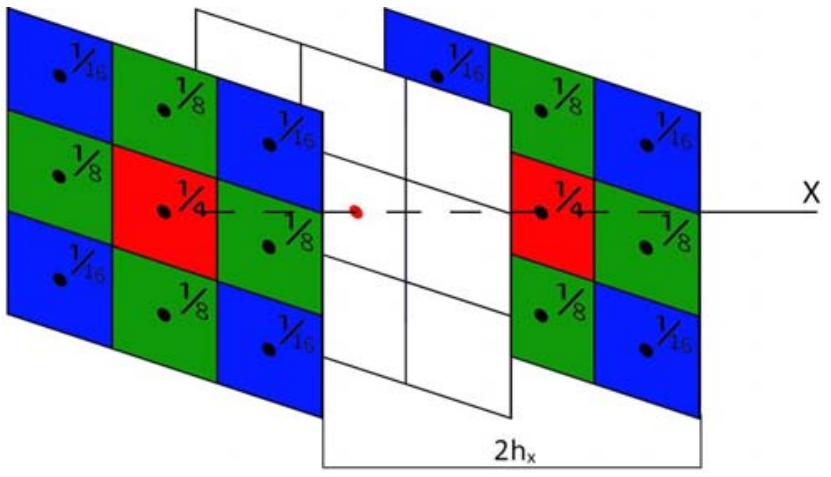

Fig. 3. Calculations of the derivative at given red point.

Formula (8) is only an approximation of the derivative. Some additional discrepancies occur also due to the limited accuracy of velocity measurements and space resolution. To estimate the possible error in the shear stress calculations we can use the fact that vectors $\vec{\tau}_{n}$ and $\vec{n}$ must be perpendicular, i.e.:

$$
\vec{\tau}_{n} \cdot \vec{n}=0
$$

Thus, the discrepancy (accuracy of calculations and measurements) measure can be taken as follows:

$$
\delta=\frac{\vec{\tau}_{n} \cdot \vec{n}}{\left|\vec{\tau}_{n}\right|}
$$

We will estimate later the accuracy of the mentioned above four methods of the derivative approximation at different moments of the cardiac cycle and time averaged values of the errors with the use of formula (11).

\section{E. User-friendly Interface}

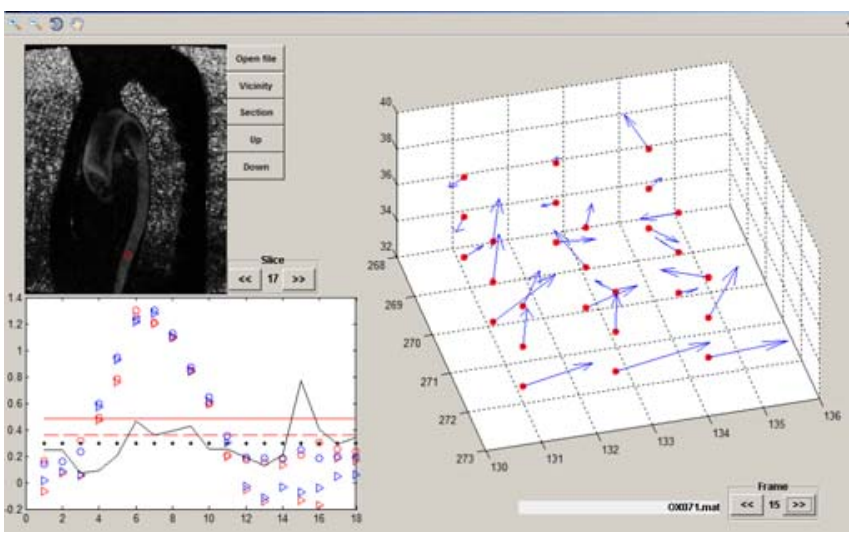

Fig. 4. The user-friendly interface. The flow velocities in $\mathrm{m} / \mathrm{s}$ and shear stresses in $\mathrm{Pa}$ (black line). Instant velocity (red markers) and averaged in time (straight red lines) and space (blue markers); the normal velocities "triangles", the magnitudes of velocities - "circles".

The calculations of the shear stress and vorticity can be carried out with the use of user-friendly interface [5], which allows selecting a point of interest (see Fig. 4 left above), blood flow visualization at this and at the neighboring 26 points (see Fig. 4 right) and displaying the values of velocities and other characteristics at different moments of time and time averaged values (see Fig. 4 right below). The interface allows changing the slices; visualizing the flow at different moment of time and displaying the time averaged values of the velocity. It can be useful to explore the flow pattern inside the vessels and in unclear areas (see Fig. 5).

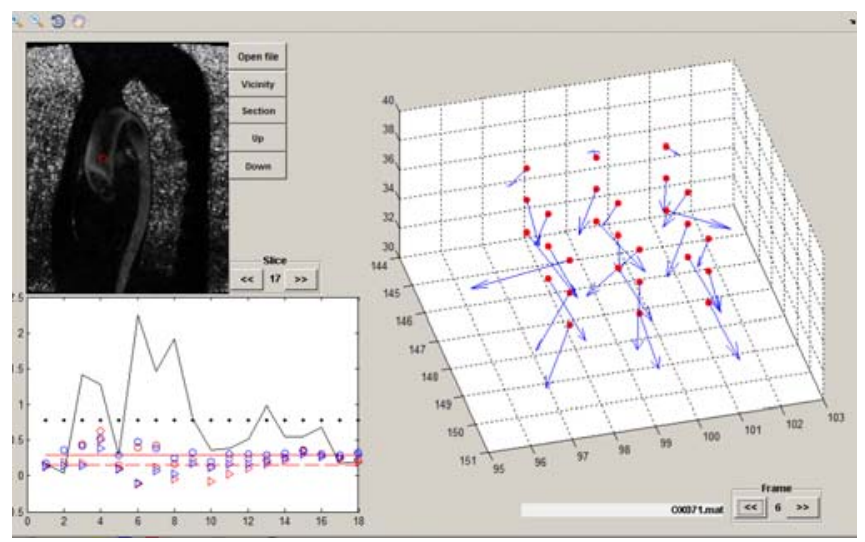

Fig. 5. The user-friendly interface. Visualization in an unclear area. The characteristics presented in the lower left graph versus time coincide with ones shown in Fig. 4.

\section{RESULTS AND DISCUSSION}

\section{A. Estimations of the Shear Stress and Its Calculation Discrepancy}

The calculations of the shear stress (formula (7)) and the discrepancy (eq.(11)) are shown in Fig. 6 (left below) for different points located inside the aorta versus moments of the cardiac cycle. Red, green, magenta and black lines and markers correspond to the differentiation methods 1-4 respectively. Lines represent the values of shear stress on the plane with the normal coinciding with the direction of the velocity in the selected point. Dotes show the time averaged values of this shear stress. The discrepancies are represented by "crosses" (instant values) and "circles" (time averaged values). It can be seen that different methods yield rather different values of the instant shear stress, but the time averaged values are close to each other. The accuracy of calculations is very limited. At some moments of time formula (11) yields the values close to +1 or -1 instead of expected close to zero figures (see eq. (10)). The time averaged values of discrepancy are also very large.

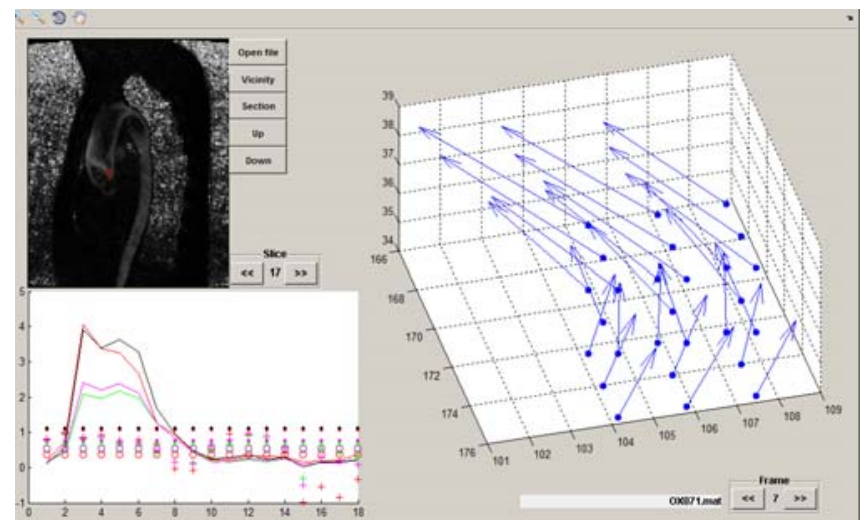



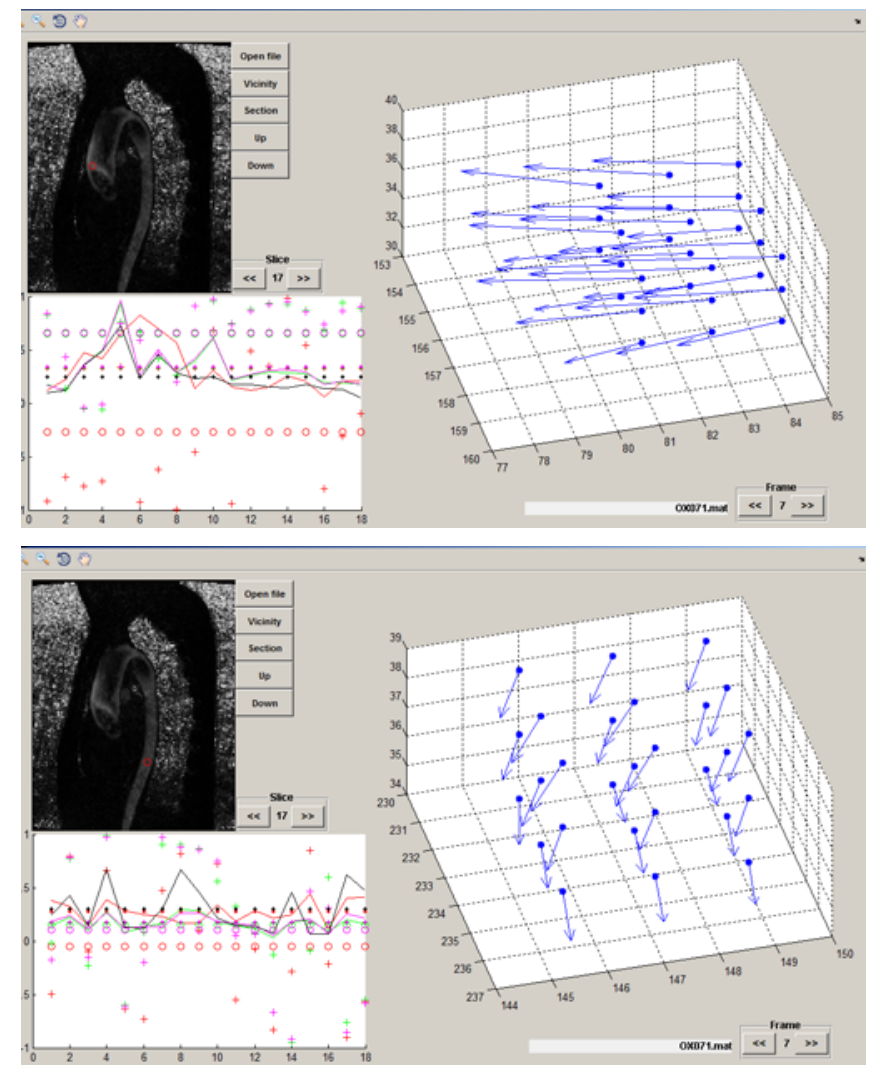

Fig. 6. Flow visualization with the use of user-friendly interface (right) in the vicinity of the selected point (left above). Shear stress and the accuracy of its calculations versus time on the plane with the normal coinciding with the direction of the velocity at the selected point (left below).

\section{B. Estimations of the Wall Shear Stress without Differentiation}

We can calculate the shear stress at any point and at any plane located in the blood flow with the use of formula (7). For medical application it is important to know this characteristic for the points located on the vessel surface, i.e., the wall shear stress (WSS). Since the accuracy and the resolution are not enough, it is useful to have some estimation of WSS, obtained without differentiation of the velocity field. Formulae (3) and (4) yield such opportunity, since the average normal velocity $u$, cross-section area and average radius can be calculated without derivatives. Black lines in Fig. 7 show the results of WSS calculations in the left ventricle, mitral valve and left atrium for patients from groups $\mathrm{C}$ and $\mathrm{A}$. It can be seen that the maximal values of the pressure gradients occur in the mitral valve and they are approximately twice smaller in the sano heart. The maximum values of the pressure gradient in the region of mitral valve does not exceed $0.001 \mathrm{~N} / \mathrm{cm}^{3}$ and cannot be a reason of a sufficient pressure drop.

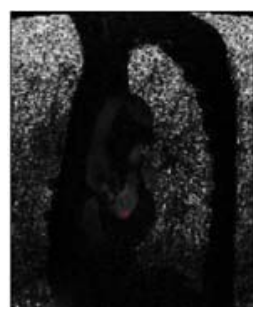

a)

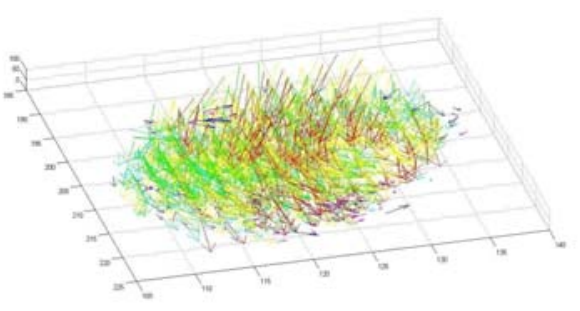

b)

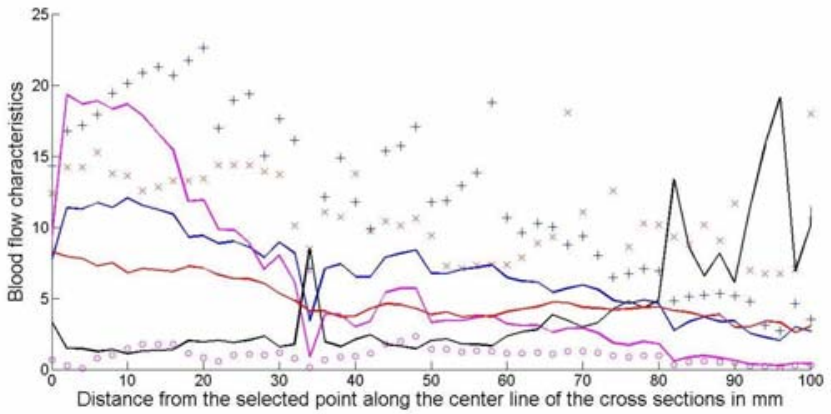

c)

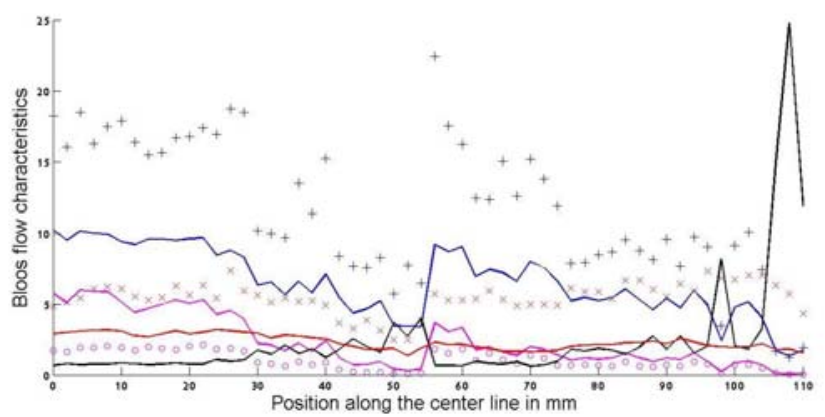

d)

Fig. 7. a) Visualization of the heart and aorta with the point selected inside the left ventricle (red circle) during diastole (time frame 13); b) The results of automatic segmentation of the points located in the left ventricle, mitral valve and left atrium. Arrows show the velocities of the blood flow at diastolic time frame 13; c) Blood flow characteristics versus distance form the selected point (shown in (a)) along the center line of the cross sections in $\mathrm{mm}$. Pressure gradient in $\mathrm{N} / \mathrm{cm}^{3}$ - black line; maximal (magenta line) and average (magenta cycles) flow rates in $1 / \mathrm{min}$; maximal (blue crosses) and average (blue line) radii of the section in $\mathrm{mm}$; maximal (red crosses) and average (red line) velocities in the section in $\mathrm{dm} / \mathrm{s}$; d) Flow characteristics (same as in Fig. 7c) in the sano heart.

Fig. 8 illustrates the increase of pressure gradient and WSS in the aortic valve for patients from group C (solid lines) in comparison with the patients from A and B groups (dotted and dashed lines). It can be seen that the ratio $u / S$ could be a good BAV detection tool even in cases when other flow parameters (in B group) are close to the normal.

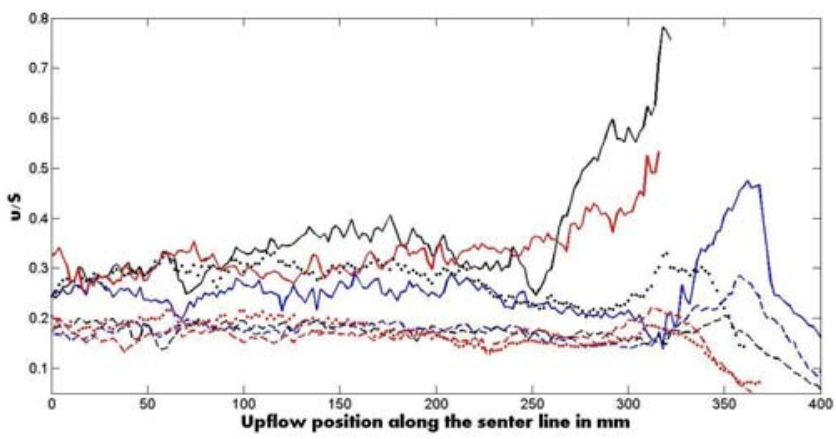

Fig. 8. Calculations of the $u / S$ ratio along the aorta and the left ventricle center line upstream from the point in descending aorta. Solid lines correspond to the $\mathrm{C}$ group (black and red lines does not show the flow in ventricles). Dotted and dashed lines correspond to patients from A and B group respectively. 


\section{Calculations of the Vorticity Vector Magnitude}

The vortexes, which occur in the unsteady separated flows and ensure a low enough pressure at their core regions, are reported as a reason of possible cavitation in MHV [10-12]. In the real mitral valves no cavitation occurs. Thus it is interesting to estimate the values of the vorticity in vivo in the mitral valves with the use of 4D MRI data. The results of calculation of the magnitude of the vorticity at different moments of the cardiac cycle are shown in Fig. 9. In the region of the mitral valve, the maximum value of $|\bar{\omega}|$ does not exceed $300 \mathrm{~s}^{-1}$ and corresponds to the moment of the valve closing (time frame 16). Closing the aortic valve of the same patient (time frame 5) causes the values of $600 \mathrm{~s}^{-1}$. The maximum magnitude of vorticity in the steady HagenPoiseuille flow equals $4 u / R$ ( $R$ is the radius of the pipe). For $u=1 \mathrm{~m} / \mathrm{s}$ and $R=10 \mathrm{~mm}$, we can have the magnitude of vorticity $400 \mathrm{~s}^{-1}$ even in the steady Hagen-Poiseuille flow. Thus, the calculated vorticity values are not enough for cavitation inception. Possibly the flexibility of the cupids of living valve prevent creating intensive vortexes and cavitation inception (in comparison with MHV).

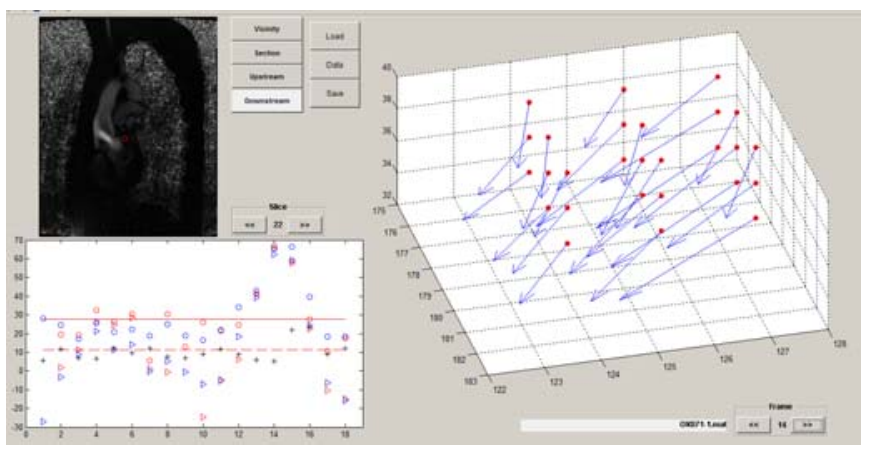

Fig. 9. Blood flow visualization with the use of user-friendly interface. The point (red circle) was selected near the mitral valve (left above). The blood flow velocities near the selected point are shown in the right window for the maximum diastolic flow rate (time frame 14). The flow velocities in $\mathrm{cm} / \mathrm{s}$ and $10 \%$ of the magnitude of the vorticity vector in $\mathrm{s}^{-1}$ (black crosses) are shown left below versus time frames: instant velocity in the selected point (red markers) and averaged in time (straight red lines) and space (blue markers); the normal velocities are presented by "triangles", the magnitudes of velocities - by "circles".

For presented $\mathrm{C}$ group patient with a bicuspid aortic valve (BAV) and dilation, the maximum detected diastolic velocity in the heart did not exceed $2 \mathrm{~m} / \mathrm{s}$. This value is not enough for cavitation, since the pressure drop $9.5 * 10^{4} \mathrm{~Pa}$ corresponds to the blood velocity $13.8 \mathrm{~m} / \mathrm{s}$. In left part of the heart of sane volunteers the maximum diastolic blood flow velocities did not exceed $1 \mathrm{~m} / \mathrm{s}$. 4D MRI data have limited space and time resolution, nevertheless the difference between the real blood velocity and one necessary for cavitation inception are too large.

\section{CONCLUSIONS}

A new accuracy measure for the shear stress was introduced to calculate the possible discrepancy which occurs due to the differentiation of the 4D MRI blood velocity field. A new software tool was developed and used to make the calculations more visible and comfortable for users. It was shown that the discrepancies could be rather high even for time averaged characteristics. To avoid differentiation, a new estimation of pressure gradient and WSS was proposed and applied for different groups of patients. The automatic segmentation of the vessels and the heart chambers yields a simple and comfortable method to identify the BAV patients even in the cases without visible blood flow changes. To estimate possible cavitation inception, the vorticity and pressure drop in mitral valve were calculated for different groups of patients.

\section{ACKNOWLEDGMENT}

The study was supported by EU-financed Horizon-2020 project AMMODIT (Grant Number MSCA-RISE 645672).

\section{REFERENCES}

[1] M. A. Bernstein et al. (2004) - Handbook of MRI Pulse Sequences, Elsevier.

[2] U. Morbiducci et al., In Vivo Quantification of Helical Blood Flow in Human Aorta by Time-Resolved Three-Dimensional Cine Phase Contrast. Annals of Biomedical Engineering, Vol. 37, No. 3, March 2009, pp. 516-531, DOI: 10.1007/s10439-008-9609-6

[3] U. Morbiducci et al. Mechanistic insight into the physiological relevance of helical blood flow in the human aorta: an in vivo study, Biomech Model Mechanobiol, DOI 10.1007/s10237-010-0238-2

[4] I. Nesteruk, A. Redaelli, I. Kudybyn, F.Piatti, F.Sturla. Global and local characteristics of the blood flow in large vessels based on 4D MRI data, AMMODIT Conference, Kyiv, Ukraine, January, 2017, Naukovi Visti NTUU KPI, 2017, No. 2, pp. 37-44, DOI: http://dx.doi.org/10.20535/1810-0546.2017.2.99724

[5] I. Nesteruk, I. Kudybyn, A. Redaelli, F. Piatti, F. Sturla, E. Votta, S.Siryk, O.Chertov, POST-PROCESSING 4D MRI IMAGES IN ORDER TO VISUALIZE THE UNSTEADY BLOOD FLOW IN LARGE VESSELS AND VENTRICLES. Biomedical engineering, № 4, April 2017. Pp. 8-11.

[6] F. Piatti, S. Pirola, M. Bissel, I. Nesteruk, F. Sturla, A. Della Corte, A. Redaelly, E.Votta. Towards the improved quantification of in vivo abnormal wall shear stresses in BAV-affected patients from 4D flow imaging: Benchmarking and application to real data, Journal of Biomechanics, 2017, v. 50 (2017), pp. 93-101.

[7] F. Piatti, F. Sturla, M. M. Bissell, S. Pirola, M. Lombardi, I. Nesteruk, A. Della Corte, A. Redaelli, E. Votta, 4D Flow Analysis of BAVRelated Fluid-Dynamic Alterations: Evidences of Wall Shear Stress Alterations in Absence of Clinically-Relevant Aortic Anatomical Remodeling. Front. Physiol., 26 June 2017, https://doi.org/10.3389/fphys.2017.00441

[8] D. G. Guzzardi et al. Valve-Related Hemodynamics Mediate Human Bicuspid Aortopathy: Insights From Wall Shear Stress Mapping. J Am Coll Cardiol. 2015 Aug 25;66(8):892-900. doi: 10.1016/j.jacc.2015.06.1310.

[9] J. R. Browning et al. 4D Flow Assessment of Vorticity in Right Ventricular Diastolic Dysfunction. Bioengineering (Basel). 2017 Apr 5;4(2). pii: E30. doi: 10.3390/bioengineering4020030.

[10] E. Rambod, M. Beizaie, M. Shusser, S. Milo, M. Gharib. 1999 A physical model describing the mechanism for formation of gas microbubbles in patients with mitral mechanical heart valves. Ann. Biomed. Eng. 27, 774-792.

[11] B. H. Maines, C. E. Brennen. 2002 Applicability of fluid transient test methods for mechanical heart valve cavitation scaling. 6th Annual Hilton Head Workshop on Prosthetic Heart Valves: Past, Present and Future, Hilton Head Island, SC, 6-10 March 2002

[12] C. E. Brennen. Cavitation in medicine, Interface Focus 5: 20150022, 2015. http://rsfs.royalsocietypublishing.org/content/5/5/20150022

[13] C. M. Zapanta et al. 1996 In vivo observation of cavitation in prosthetic heart valves. ASIAO J. 42, M550-M554. (doi:10.1097/00002480-199609000-00047)

[14] L. G. Loitsyanskiy, Mechanics of Liquids and Gases, Begell House, New York and Wallingford, 6th ed, 1995, $961 \mathrm{p}$.

[15] B. Jähne, H. Scharr, and S. Körkel. Principles of filter design. In Handbook of Computer Vision and Applications. Academic Press, 1999. 